\title{
Use of high-density EEG in patients with Parkinson's disease treated with deep brain stimulation
}

\author{
Jiri Burila,b, Petra Burilovac, Andrea Pokornac, Marek Balaz ${ }^{a, b}$
}

\begin{abstract}
The number of reports on cognitive changes related to deep brain stimulation (DBS) surgery in Parkinson's disease (PD) is growing. The aim of the review was to assess whether high-density electroencephalography (HD EEG) in patients with DBS treatment for PD is used and mentioned as a research method for the assessment of cognitive function changes after DBS of the subthalamic nucleus. The HD EEG examination method demonstrates the effects of DBS on changes of connectivity among various areas of the brain using changes in bioelectric potentials recorded on the scalp during examination. The search results yielded seven studies on the possibility of using HD EEG to investigate changes in bioelectric potentials with various protocols (motor tasks, auditory stimuli). Based on the results, HD EEG appears to be a feasible research method in patients with PD and established DBS therapy. Therefore, we plan to use HD EEG to demonstrate the effects of DBS on changes of connectivity among various areas of the brain utilizing changes in bioelectric potentials recorded on the scalp during HD EEG examination.
\end{abstract}

Key words: Parkinson's disease, deep brain stimulation, high density, electroencephalography, cognitive changes

Received: November 5, 2019; Revised: July 14, 2020; Accepted: September 15, 2020; Available online: September 21, 2020

https://doi.org/10.5507/bp.2020.042

(c) 2020 The Authors; https://creativecommons.org/licenses/by/4.0/

a1st Department of Neurology, St. Anne's University Hospital, Brno, Czech Republic

${ }^{b}$ Department of Neurology, Faculty of Medicine, Masaryk University, Brno, Czech Republic

'Department of Nursing and Midwifery, Faculty of Medicine, Masaryk University, Brno, Czech Republic

Corresponding author: Jiri Buril, e-mail: Jiri.Buril@fnusa.cz

\section{INTRODUCTION}

Deep brain stimulation (DBS), one of the treatment methods primarily used for movement disorders, is gradually beginning to emerge in the treatment of pharmacoresistant epilepsy and obsessive-compulsive disorder ${ }^{1}$. DBS is now a commonly used neuromodulatory therapy for late motor complications in patients with advanced Parkinson's disease (PD). This method is widely represented at this stage of the disease, in appropriately indicated patients. Tens of thousands of PD patients have been treated with DBS since the 1990s. DBS is indicated in patients with advanced motor complications of PD who are severely restricted in routine daily activities and who developed levodopa-related adverse effects. Surgery may be risky for the elderly and for patients who experience psychiatric symptoms or cognitive disorders related or unrelated to PD (ref. ${ }^{2}$ ). The subthalamic nucleus (STN) appears to be the most frequent target for DBS in PD. While STN DBS alleviates cardinal motor symptoms of PD (namely tremor, rigidity and bradykinesia) and improves the quality of life of PD patients, several reports have shown that this treatment may be related to worsening of cognitive functioning ${ }^{3,4}$. A multidisciplinary team (neurosurgeon, neurologist, neuropsychologist, neuroradiologist) should be involved in decision-making on the indication of the procedure itself 5 .

STN DBS may have an impact on cortical functions as shown by its effect on scalp EEG or long-latency evoked potentials ${ }^{6}$. However, classical 19-channel EEG may not be sufficient to delineate discrete changes in cortical bioelectric potentials due to the low number of electrodes over cortical areas.

Therefore, the use of high-density EEG (HD EEG) may be warranted as it uses 256 channels and provides more detailed coverage and mapping of cortical activity ${ }^{7}$. So, based on the above evidence, we plan to use HD EEG to anticipate changes in cerebral cortex activity related to STN DBS stimulation ${ }^{8}$. The results of HD EEG examination will be further processed and will help to create a cortical map of bioelectric potentials with a possibility to evaluate changes of connectivity, both between individual areas of the cerebral cortex, or in relation to neurostimulation in PD patients treated with DBS (comparison of states with DBS stimulation turned on and off), as already proven ${ }^{9}$. Comparison of results of these examinations may allow us to assess the effect of DBS on cortical functions by demonstrating a change in connectivity. As the first logical step of our research, we performed a literature review.

\section{METHODOLOGY}

The PubMed, Scopus, and Web of Science databases were used for the search of scientific articles.

All searches were dated from January 2010 until August 2019. 


\section{RESULTS}

The database searches identified 1659 original articles (PubMed 182; Scopus 766; Web of Science 711) (Fig. 1). Another source was the Epistemonikos database; however, with no findings of relevant existing reviews or ongoing studies.

Article references were stored in EndNote Web and duplicate records were removed. Subsequently, the abstracts or full texts of the remaining articles were checked to exclude records that did not meet the criteria for inclusion in the literature review. Articles meeting the inclusion criteria after reviewing the abstracts or full texts were thoroughly re-evaluated to ensure their suitability for the literature review. The flowchart in Fig. 1 shows the process of obtaining the required records.

Records that did not meet the requirements and criteria for an original article ${ }^{10}$ were excluded. In general, only articles focusing on PD were considered. Finally, based on search results, seven studies using EEG methods to predict cognitive status (deterioration/ improvement) were included (Table 2).

\section{DISCUSSION}

The aim of this review was to verify the use of HD EEG in patients with PD and to briefly describe methodology used in the evaluated articles. The first reviewed study examined a new method for removing DBS artifacts from EEG. This study was chosen because of the need to modify obtained data which are degraded and unreadable due to artifacts during active DBS. Based on this research, further processing of HD EEG / EEG data in PD patients and established DBS therapy is possible ${ }^{11}$. Various neurophysiological methods are used in research. In our study we selected HD EEG. The method and its advantages were described in a study focusing on differences

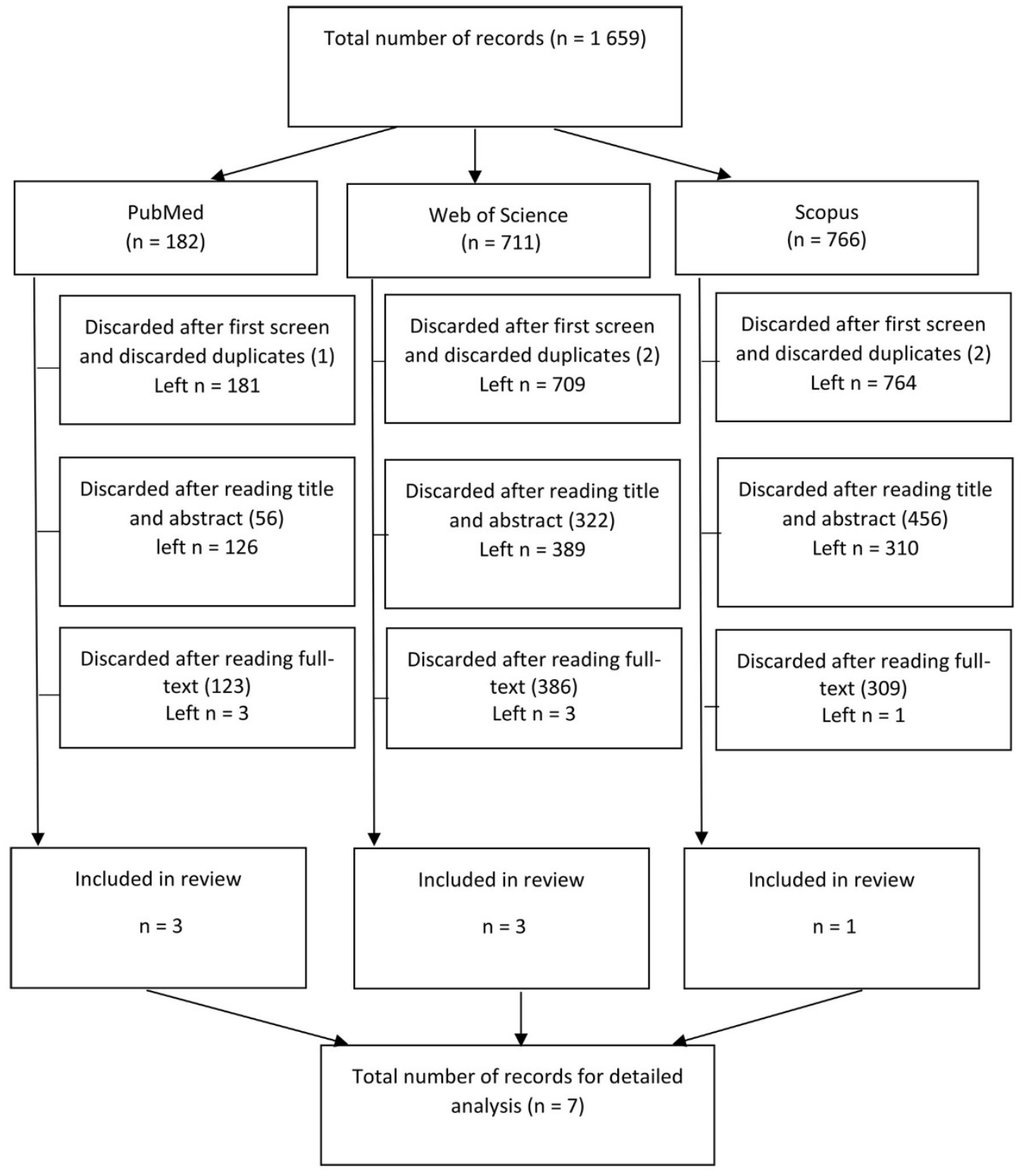

Fig. 1. Flow chart. 
Table 1. Inclusion and exclusion criteria for the literature review.

\begin{tabular}{ll}
\hline Inclusion criteria & Exclusion criteria \\
\hline Deep brain stimulation treatment & Low number of EEG channels* \\
Use of HD EEG & Another movement disorder \\
Cognitive changes & \\
Diagnosis G20 - Parkinson's disease (ICD-10) & \\
No age limit for the study population & \\
\hline
\end{tabular}

*A low number of channels means the use of a standard EEG system with 19 or fewer channels.

Table 2. Summary - studies included in the final evaluation.

\begin{tabular}{|c|c|c|c|c|c|c|c|c|c|}
\hline Authors & Year & Country & $\begin{array}{l}\text { Type of } \\
\text { study }\end{array}$ & $\begin{array}{l}\text { Size of the } \\
\text { sample (control/ } \\
\text { intervention) }\end{array}$ & $\begin{array}{l}\text { Diagnosis of } \\
\text { the sample }\end{array}$ & $\begin{array}{l}\text { DBS } \\
\text { treat- } \\
\text { ment }\end{array}$ & $\begin{array}{l}\text { HD } \\
\text { EEG }\end{array}$ & $\begin{array}{l}\text { No. of EEG } \\
\text { channels }\end{array}$ & Ref. \\
\hline Perfetti B et al. & 2010 & USA/ Italy & Prospective & 19 & G20 & no & yes & 256 & 13 \\
\hline Herz DM et al. & 2012 & $\begin{array}{l}\text { Germany/ } \\
\text { Denmark/ Canada }\end{array}$ & Prospective & $11 / 13$ & G20 & yes & yes & 122 & 22 \\
\hline Sun Y et al. & 2014 & Canada & Prospective & 15 & $\mathrm{~N} / \mathrm{A}^{*}$ & yes & no & $19 * *$ & 11 \\
\hline Klamer S et al. & 2015 & Germany/ Italy & Prospective & 14 & $\mathrm{~N} / \mathrm{A}^{*}$ & no & yes & 256 & 12 \\
\hline Gulberti A et al. & 2015 & Germany & Prospective & $12 / 12$ & G20 & yes & yes & 128 & 14 \\
\hline Gulberti A et al. & 2015 & Germany & Prospective & $12 / 12$ & G20 & yes & no & 62 & 18 \\
\hline Markser A et al. & 2015 & Germany & Prospective & 30 & G20 & yes & no & $20 * *$ & 17 \\
\hline
\end{tabular}

Note: the results of 7 studies that met all the search criteria and their full texts were found in the databases.

*Patients with PD were not directly studied, but the authors virtually modelled DBS artifacts and illustrated the removal technique on EEG data.

**Despite the number of EEG channels defined in the exclusion criteria, two studies with 19 and 20 channels were included due to the overall low number of records found.

between magnetoencephalography (MEG) and HD EEG in signal resource localization using a distributed source model compared to functional magnetic resonance imaging (fMRI). The results supported the use of HD EEG rather than MEG for changes in source localization ${ }^{12}$.

Due to the relatively low number of publications with research focus similar to our planned research we also assessed articles that used similar methodology and neurophysiological approaches and, with the same group of subjects examined, we also included records with PD patients and established DBS therapy. Using this criterion, we found an HD EEG study focusing on modulation of attention regulating motor and non-motor performance in PD patients ${ }^{13}$. According to the study, attention processes that share neural resources with those underlying the spatial sequential learning and working memory support early response to unpredictable stimuli.

A similar study investigated whether STN DBS in patients with PD improved sensory perception. The influence of levodopa and STN DBS on auditory processing was studied by rhythmic auditory stimulation (RAS) with subsequent sensing of auditory evoked potentials ${ }^{14}$. The possibility for use of perioperative EEG in patients undergoing DBS surgery with the aim of determination of recorded scalp signals topography was formulated ${ }^{15}$.

Possible use of EEG in patients with PD was also reported in a study with levodopa that aimed to restore connectivity from the prefrontal to premotor cortex during externally stimulated movement in PD patients ${ }^{16}$. Cortical activity was observed with HD EEG in PD subjects who repeatedly performed a motor task. Another group of authors considered a possible predictive use of the EEG method in assessment of preclinical cognitive deterioration in patients with PD and established DBS therapy. This research was mainly focused on possible negative effects of DBS on cognitive functioning ${ }^{17}$.

The last relevant study focused on the predictive timing functions of cortical beta oscillations which are impaired in PD and may be affected by levodopa and DBS. Primarily, the effect of levodopa and STN DBS on resting state EEG and on the cortical response profile to slow and rapid RAS in a passive listening paradigm focusing on beta band oscillations important for auditory-motor connections was studied ${ }^{18}$. The main evidence of this method's effectiveness was description of the predictive value of HD EEG use in patients with PD by performing pre-operative EEG and one post-operative EEG examination $^{19}$. For EEG analysis, two experienced neurologists who independently used the Grand Total EEG score (GTE-TS) (ref. ${ }^{20}$ ) and then a short version of the Grand Total EEG score (GTE-SS) (ref. ${ }^{21}$ ) for visual evaluation of EEG pathologies were required. The results were used to assess cognitive decline. Significant increases in GTE-SS and GTE-TS after DBS implantation were observed in the entire group of patients. In a total of 26 patients undergoing EEG examinations in two follow-up tests (FU-1 and FU-2), alpha-corrected post hoc Wilcoxon tests showed significant GTE-SS and GTE-TS elevations on FU-1 and FU-2 compared to preoperative scores. In comparison with later research using HD EEG, they used a system with 122 channels $^{22}$. EEG data were processed with the BESA software. By using fMRI they defined the area of interest, namely motor cortical networks ${ }^{23}$. This cortical network contained the primary motor cortex (M1), pre- 
motor areas (PM) and supplementary motor area (SMA) in the left hemisphere. Then the frontoparietal cortical region was defined using these data. The same procedure was also performed for the second region of interest in the left prefrontal cortex (PFC). In both patients and control subjects, EEG data connectivity analysis was performed using dynamic causal modelling (DCM) for induced responses implemented in the SPM8 software ${ }^{24}$. DCM was based on a modelling framework that included a motor cortical network containing left M1, PM and SMA as well as left PFC. As a result of this study, the interactions between M1, PM and SMA and the interactions between PFC and PM as well as PFC and SMA were assumed. Since the aim of this study was to assess the effective connectivity between prefrontal and premotor regions, the analysis focused on the oscillatory links in the reciprocal PFC-M1 and PFC-SMA connections. Correlation analysis was performed for each participant; individual binding values were extracted from the bonds between the prefrontal and premotor regions, which were significantly modulated by motor action during the task.

\section{CONCLUSION}

All the studies found in the literature review used an EEG method. However, they differed in the exact type of system used and in the particular EEG / HD EEG methodology. The results of the above studies present the possibility of using this method to investigate changes in bioelectric potentials with various protocols (motor tasks, auditory stimuli) as well as to influence these bioelectric potentials using a therapeutic neuromodulation method, in this case DBS.

Based on the proven effective use of HD EEG in patients with PD and established DBS therapy, we plan to use this method to demonstrate the effects of DBS on cognitive functions that may be based on connectivity changes in various areas of the brain related to changes in bioelectric potentials during HD EEG examination.

\section{Search strategy and selection criteria}

The review was conducted in English based on a clinical question with the following search terms and operators: "Parkinson's disease" AND "deep brain stimulation" OR “DBS” AND "HD EEG” OR "high-density electroencephalography" AND “cognitive changes". As the search yielded no original articles, the strategy was narrowed to "Parkinson's disease" AND "deep brain stimulation" OR "DBS".

The following data were extracted from selected articles: size of the sample, deep brain stimulation treatment, use of an EEG method to predict cognitive change (deterioration/improvement) and number of EEG channels (Table 1).

\section{ABBREVIATIONS}

DBS, Deep brain stimulation; DCM, Dynamic causal modelling; fMRI, functional magnetic resonance imaging; FU, Follow-up; GTE-TS, Grand Total EEG, total score; GTE-SS, Grand Total EEG, short score; HD EEG, High-density electroencephalography; ICD-10, International Statistical Classification of Diseases and Related Health Problems; M1, Primary motor cortex; MEG, Magnetoencephalography ; PD, Parkinson's disease; PFC, Prefrontal cortex; PM, Premotor areas; RAS, Rhythmic auditory stimulation; SMA, Supplementary motor area; STN, Subthalamic nucleus.

Acknowledgement: None declared.

Author contributions: JB: manuscript writing and conducted the literature review; PB, AP, MB: provided broad ideas and structure, as well as revisions; JB, PB, AP: concept and design, data collection and review; JB, PB: manuscript draft; $\mathrm{MB}$, AP: critical revision of the manuscript and final approval of the manuscript.

Conflict of interest statement: The authors declare no conflict of interest. The review was carried out as part of a dissertation thesis at the Masaryk University.

\section{REFERENCES}

1. Temel Y, Jahanshahi A. Treating brain disorders with neuromodulation. Neuroscience 2015;347:1418-19.

2. Chunyan C, Dianyou L, Tianxiao, J, Nuri Fl, Shikun Z, Jing Z, Zhiyi S, Bomin S. Resting State Cortical Oscillations of Patients With Parkinson Disease and With and Without Subthalamic Deep Brain Stimulation. J Clin Neurophysiol 2015;32(2):109-18. doi: 10.1097/ WNP.0000000000000137

3. Katlowitz KKoM, Mogilner AY, Pourfar M. Effect of deep brain simulation on arm, leg, and chin tremor in Parkinson disease. J Neurosurg 2018;1:1-6. doi: 10.3171/2018.7.JNS18784

4. Jech R, Růzička E, Urgosík D, Serranová T, Volfová M, Nováková $O$, Roth J, Dušek $P$, Mečír $P$. Deep brain stimulation of the subthalamic nucleus affects resting EEG and visual evoked potentials in Parkinson's disease. Clin Neurophysiol 2006;117(5):1017-28.

5. Koeglsperger T, Palleis C, Hell F, Mehrkens JH, Bötzel K. Deep Brain Stimulation Programming for Movement Disorders: Current Concepts and Evidence-Based Strategies. Front Neurol 2019;10:410. doi: 10.3389/fneur.2019.00410

6. Kovacs N, Balas I, Kellenyi L, Janszky J, Feldmann A, Llumiguano C, Doczi TP, Ajtay Z, Nagy F. The impact of bilateral subthalamic deep brain stimulation on long-latency event-related potentials. Parkinsonism Relat Disord 2008;14(6):476-80. doi: 10.1016/j.parkreldis.2008.01.002

7. Odekerken VJ. GPi versus STN deep brain stimulation for Parkinson disease: three-year follow-up. J Neurology 2016;86:755-61.

8. Herzog J, Fietzek U, Hamel W, Morsnowski A, Steigerwald F, Schrader B, Weinert D, Pfister G, Müller D, Mehdorn HM, Deuschl G, Volkmann J. Most effective stimulation site in subthalamic deep brain stimulation for Parkinson's disease. Mov Disord 2004;19:1050-54. doi: 10.1002/mds.20056

9. Eisinger RS, Ramirez-Zamora A, Carbunaru, S, Ptak B, Peng-Chen Z, Okun MS, Gunduz A. Medications, Deep Brain Stimulation, and Other Factors Influencing Impulse Control Disorders in Parkinson's Disease. Frontiers 2019;10:86. doi: 10.3389/fneur.2019.00086

10. Sollaci LB, Pereira MG. The introduction, methods, results, and discussion (IMRAD) structure: a fifty-year survey. J Med Libr Assoc 2014;92(3):364-71.

11. Sun $Y$, Farzan F, Garcia Dominguez L, Barr MS, Giacobbe $P$, Lozano AM, Wong W, Daskalakis ZJ. A novel method for removal of deep 
brain stimulation artifact from electroencephalography. J Neurosci Methods 2014;237:33-40. doi: 10.1016/j.jneumeth.2014.09.002

12. Klamer S, Elshahabi A, Lerche $H$, Braun Ch, Erb M, Scheffler $K$ Focke NK. Differences Between MEG and High-Density EEG Source Localizations Using a Distributed Source Model in Comparison to fMRI. Brain Topogr. 2015;28(1):87-94. doi: 10.1007/s10548-014-04053

13. Perfetti B, Moisello C, Lanzafame $S$, Varanese $S$, Landsness EC, Onofrj M, Rocco ADi, Tononi G, Ghilardi MF. Attention modulation regulates both motor and non-motor performance: a high-density EEG study in Parkinson's disease. Arch italiennes de biologie 2010;148(3):27988.

14. Gulberti A, Hamel W. Subthalamic deep brain stimulation improves auditory sensory gating deficit in Parkinson's disease. Clin Neurophysi 2015;126(3):565-74. doi: 10.1016/j.clinph.2014.06.046

15. Hilker R, Benecke R, Deuschl G, Fogel W, Kupsch A, Schrader C, Sixel-Doring F, Timmermann L, Volkmann J, Lange M. Deep brain stimulation for Parkinson's disease. Consensus recommendations of the German Deep Brain Stimulation Association. Nervenarzt 2009;80:646-55.

16. Herz DM, Siebner HR, Hulme OJ, Florin E, Christensen MS, Timmermann L. Levodopa reinstates connectivity from prefrontal to premotor cortex during externally paced movement in Parkinson's disease. Neurolmage 2014;15(90):15-23. doi:10.1016/j.neuroimage.2013.11.023

17. Markser A, Maier F, Lewis CJ, Dembek TA, Pedrosa D, Eggers $C$ Timmermann L, Kalbe E, Fink GR, Burghaus L. Deep brain stimulation and cognitive decline in Parkinson's disease: The predictive value of electroencephalography. J Neuology 2015;262(10):2275-84. doi: $10.1007 / 500415-015-7839-8$
18. Gulberti A, Moll CK, Hamel W, Buhmann C, Koeppen JA, Boelmans K, Zittel S, Gerloff C, Westphal M, Schneider TR, Engel AK. Predictive timing functions of cortical beta oscillations are impaired in Parkinson's disease and influenced by L-DOPA and deep brain stimulation of the subthalamic nucleus. Clin Neurolmage 2015;9:436-49. doi:10.1016/j.nicl.2015.09.013

19. Morita A, Kamei S, Mizutani T. Relationship between slowing of the EEG and cognitive impairment in Parkinson disease. J Clin Neurophysiol 2011;28:384-87.

20. De Gaspari D, Siri C, Landi A, Cilia R, Bonetti A, Natuzzi F, Morgante L, Mariani CB, Sganzerla E, Pezzoli G, Antonini, A. Clinical and neuropsychological follow up at 12 months in patients with complicated Parkinson's disease treated with subcutaneous apomorphine infusion or deep brain stimulation of the subthalamic nucleus. $J$ Neurology Neurosurgery Psychiatry 2009;77:450-53.

21. Klassen BT, Hentz JG, Shill HA, Driver-Dunckley E, Evidente VG, Sabbagh MN, Adler CH, Caviness JN. Quantitative EEG as a predictive biomarker for Parkinson disease dementia. J Neurology 2011;77:11824.

22. Herz DM, Christensen MS, Reck CH, Florinade E. Task-specific modulation of effective connectivity during two simple unimanual motor tasks: A 122-channel EEG study. J Neurolmage 2012;59(4):3187-93.

23. Haslinger B, Erhard P, Kampfe N, Boecker H, Rummeny E, Schwaiger $M$. Event-related functional magnetic resonance imaging in Parkinson's disease before and after levodopa. J Brain 2010;124:55870.

24. Chen CC, Kilner JM, Friston KJ, Kiebel SJ, Jolly RK, Ward NS. Nonlinear coupling in the human motor system. J Neuroscience 2010;30:839399. 